\title{
The propagation and accumulation of dimensional shrinkage in investment casting
}

\author{
Bo $\mathrm{He}^{1, \mathrm{a}}$, Donghong Wang ${ }^{2, \mathrm{~b}}$, Fei $\mathrm{Li}^{1, \mathrm{c}}$, Heping $\mathrm{Lv}^{1, \mathrm{~d}}$ \\ ${ }^{1}$ State Key Laboratory of Metal Matrix Composites, Shanghai Jiao Tong University, Shanghai, \\ 200240, China \\ ${ }^{2}$ School of Materials Engineering, Shanghai University of Engineering Science, Shanghai, \\ 201620, China

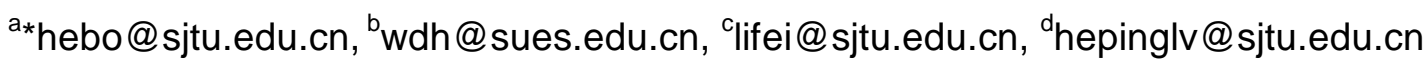

\begin{abstract}
Keywords: dimensional shrinkage; wax pattern; investment casting; propagation and accumulation. Abstract. The injection of wax pattern and the solidification of the metal are both essential procedures and having the major influences on the final dimensions. However, the main casting process steps and casting geometry affecting the dimensional shrinkage wasn't quantitatively studied. In this study, dimensional shrinkage has been investigated with a ring to ring part. The displacement of wax pattern and its corresponding casting is measured by 3D laser scanner. An unconstrained dimension of diameter is, on average, contract with $0.849 \%$ for wax pattern and $2.559 \%$ for casting. A direct linear relationship of dimensions in outer ring is established between the wax patterns with its castings. The statistical quantitative results show that the accuracy of dimensions in outer ring is dominantly influenced by metal thermal contraction in casting process.
\end{abstract}

\section{Introduction}

Typical of aero engine cartridge receiver, which consists of barrel-shaped or ring to ring structure, are some of the critical components of an aero engine. These components, which have close dimensional and geometrical tolerances, are manufactured by the investment casting process. However, there are a number of dimensional systematic errors affect the amount of dimensional changes of this casting, including four major dimensional changes associated: tooling machining tolerances, shrinkage of wax pattern, expansion of shell and shrinkage of alloy [1-3]. The fundamental physical shrinkage of the die-to-wax and the casting solidification were the two main reasons for the final casting dimensional changes [4]. Studies [5] show that the dimension accuracy of the final casting is mainly propagated from its corresponding wax pattern in steel casting.

Current methods of producing dimensionally accepted castings were choosing the proper pattern dimensional allowances. Thus, it becomes important to understand and control of the factors that affect the shrinkage of the final cast. Much effort has been done to obtain the exact pattern dimensional shrinkage [6-8]. Some foundries used the constant tooling shrinkage allowances for the entire casting regardless of casting size or feature geometry [6]. Others used the specific tooling allowances in casting directions neglecting the structure types of the casting and constraint conditions of the shell mould [7]. Different pattern shrinkage values were surveyed in wax pattern for free, fully constrained, and partially restrained feature dimensions [8]. It takes a lot of trouble to choose the shrinkage allowance for dimensions less than $25.4 \mathrm{~mm}$ in steel castings. To date, the numerical simulation method is used by industry to predict the dimensional changes occurring during solidification and cooling. Although there are some valuable guidelines for establishing allowances for one type of wax, one type of metal and one post-treatment process. The dimensional transfer relationship of wax pattern to its corresponding shell and casting is still obscure. In order to obtain more realistic and quantitative results, the main casting process steps and casting geometry affecting the dimensional shrinkage must be quantified and evaluated. 
The aims of this work are to develop further understanding of the processes and specific features contribute to the dimensional shrinkage of the casting in investment casting. The relationship of dimensional shrinkage between the wax pattern and its corresponding casting was also investigated.

\section{Experimental work}

A ring to ring part with four connect plates was designed similarity and used in the assessment of dimensional accuracy of wax pattern and casting, as shown in Figure 1(a). There were five steps in the outer ring with different stepped thickness of 2, 4, 8, 16,24mm. The maximum diameter in outer ring was $348 \mathrm{~mm}$. Firstly; the wax pattern of KC4017B was produced and assembled with spur systems. Secondly; the ceramic shell mould were made from multi-component slurries, including fine mesh refractory fillers and colloidal binder system, as shown in Figure 1(b). Thirdly, the shell was exposed to steam autoclaving at $180^{\circ} \mathrm{C}$ with a high pressure. Then, the shell was heated to $1000^{\circ} \mathrm{C}$ for $4 \mathrm{~h}$ in a vacuum casting furnace. Finally, the Ni-based superalloy K4169 was melted in a vacuum investment casting system, and then poured into gating system under gravity. The final casting dimensions were measured by FARO laser scanner, as shown in Figure 1(c). The $450 \mathrm{kv}$ industrial computerized tomography with $\pm 8 \mu \mathrm{m}$ accuracy was applied to measure the inner cavity dimensions of the ceramic shell which has been sintered.

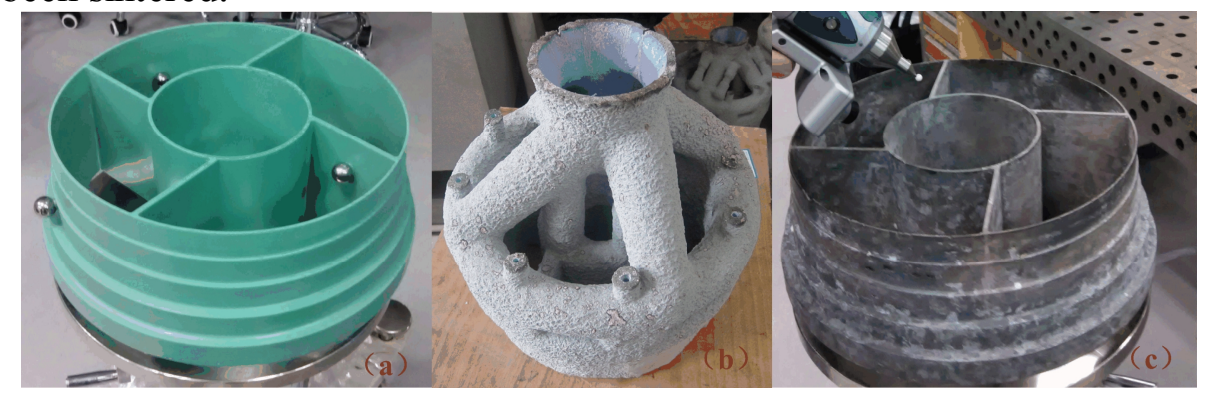

Figure 1. Geometry of the ring to ring part used in this study.(a) Wax pattern,(b) Ceramic shell, (c) K4169 superalloy casting

We can analysis the error of the wax pattern and casting from the average error deviation between theoretical model and scanning point clouds [8]. In addition, we can also compare the same cross section contour, calculating the distance and analysing the error. Though using $2 \mathrm{D}$ comparison command, we compare the theoretical model with the scanning model, which was cut by system section plane along the contour line with different rotate direction $\left(30^{\circ}, 60^{\circ}, 120^{\circ}\right.$, and $\left.150^{\circ}\right)$. The diameter and thickness values were average of the four section contour positions dimensions. The analysing result cut by the system section plane is shown in Figure 2. The casting dimensions of scanned points clouds is shown in Figure 2(a), with the deviation colour spectrum of dimension for wax pattern is shown in Figure 2(b). Shrinkage of the wax pattern and casting were calculated with the following equation as where $\mathrm{L}_{0}$ is the design pattern dimension in the mould cavity (original design dimension) and $\mathrm{L}$ is the dimension of corresponding wax pattern, the inner cavity of shell and casting.

Linear shrinkage $=\left(\mathrm{L}_{0}-\mathrm{L}\right) / \mathrm{L}_{0} \times 100 \%$.
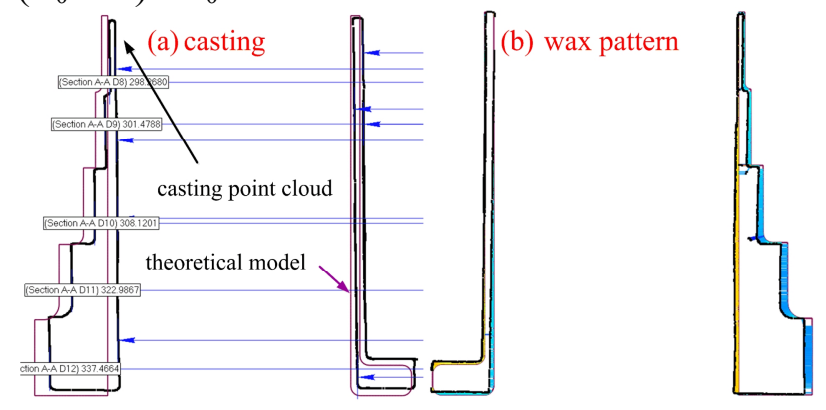

Figure 2. 2D cross section contour comparison between scanned part and the original model (a) K4169 superalloy casting, (b) Wax pattern. 


\section{Free shrinkage behavior of wax pattern and casting}

Some researchers have defined the outer ring diameter and thickness as unconstrained dimensions of the wax pattern and casting. The shrinkage variation of the outer ring diameter for the wax pattern and casting as a function of pattern dimension is shown in Figure 3. Clearly, there are typical different shrinkage values for castings and wax patterns under free shrinking conditions. The dimensional shrinkage of castings as a function of feature dimensions in outer ring diameter followed the same trends as those for wax pattern. With the increased of the diameter, the shrinkage of wax pattern and casting increased synchronously. The casting in the outside ring cools faster because they were surrounded by cool air, which resulted in lager dimensional shrinkage in casting process. The wax pattern was solidified instantly formed a relatively small part shrinkage in total casting process. As the K4169 superalloy casting cools from pouring temperature of $1500^{\circ} \mathrm{C}$, it contracts from liquid to solid with a large volume change. Solid contraction of the metal is the main influence factors determine the final dimension shrinkage of the casting because of liquid metal feeding for solidification. The shrinkage rule of casting in diameter was derived from the corresponding wax pattern. The diameter of the outer ring is, on average, contract with $0.849 \%$ for wax pattern and $2.559 \%$ for casting. As we can see from Figure 4, the values of contraction of thickness was very large than that of the diameter in outer ring. The shrinkage of thickness $4 \mathrm{~mm}$ is larger than others. The reasons for this are not immediately clear but might have some connections with the influence of the neighbouring features. An increase of the thickness from 4 to $24 \mathrm{~mm}$ results in a shrinkage decrease from $4.28 \%$ to $3.46 \%$, for the wax pattern. The shrinkage of wax pattern was conditioned at different cavity pressure and cooling history resulting litter difference in shrinkage in thickness. It demonstrated the difficulty of determining the pattern shrinkage for the casting thickness because of gating and runner system feeding. It is suggested that different dimension type and size hold different pattern shrinkage allowances and dimensional tolerances.

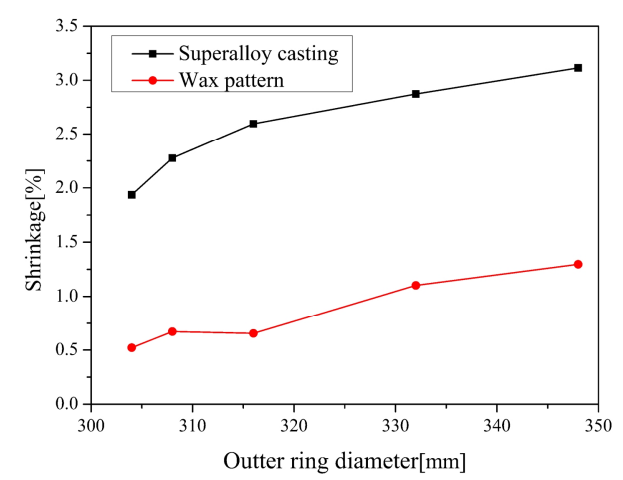

Figure 3. Shrinkage of diameter in outer ring for wax pattern and casting

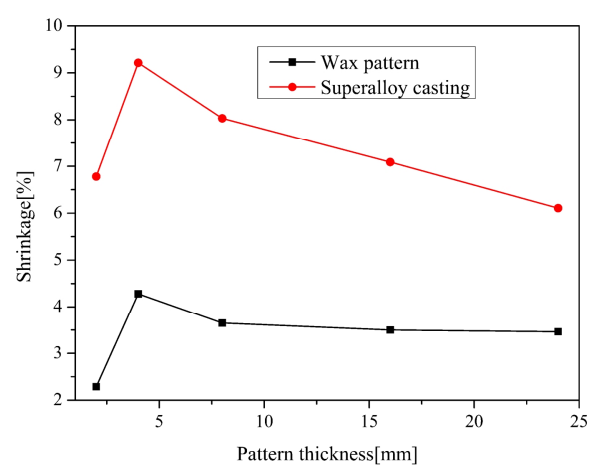

Figure 4. Shrinkage of thickness in outer ring for wax pattern and casting 


\section{The relationship between wax pattern and casting in dimensions}

There is no definite dimensions relationship between the wax pattern and its casting. The traceability of the dimensional accuracy in all casting process was evaluated. It was possible to trace individual casting dimensions to its wax pattern dimensions, as shown in Figure 5. The wax pattern gives a linearly proportional of $98.362 \%$ to the corresponding K4169 casting in the diameter of outer ring. A direct linear relationship in thickness is also found between wax pattern and its casting in outer ring, as shown in Figure 6. The thickness of wax pattern was found to be about 3.187\% larger than the casting thickness in outer ring. The above data suggests that the dimensional changes of wax pattern must be carefully controlled in order to produce high precision castings.

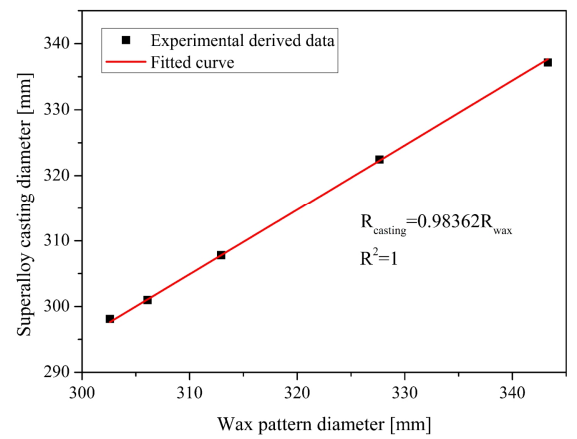

Figure 5. Relationship of diameter in outer ring between the wax pattern and its corresponding casting.

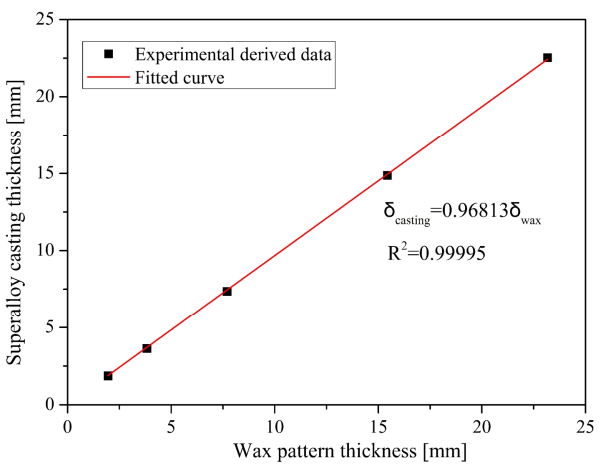

Figure 6. Relationship of thickness in outer ring between the wax pattern and its corresponding casting.

\section{The effect of process on dimensional shrinkage}

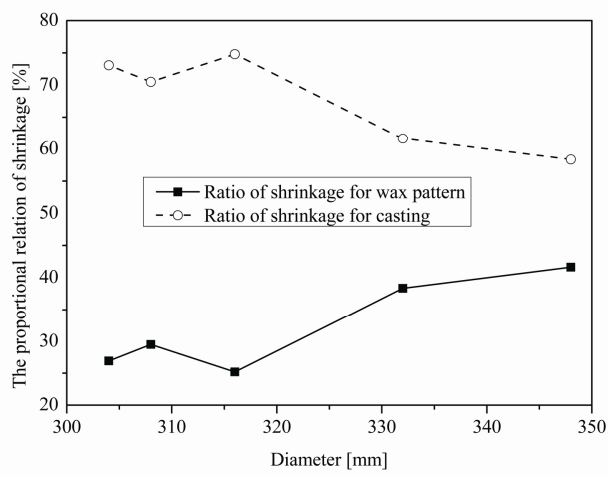

Figure 7. The proportional relation of shrinkage in diameter between wax pattern and its corresponding casting 
As we can see from the Figure 7, the effect of wax pattern process on final casting dimensions was variation in the range from $25.2 \%$ to $41.6 \%$ for diameter in outer ring. The proportion of shrinkage of outer ring diameter for wax pattern was less than $50 \%$ in total casting dimensional changes. We can conclude that the dimensional accuracy of castings in all casting processes is dominantly influenced by that of dimension changes in casting thermal contraction stage. The shrinkage of alloy is the main factor of the overall dimensional changes between the pattern tooling and its corresponding K4169 superalloy casting.

\section{Conclusions}

The wax pattern and casting can shrinkage freely at the unconstrained dimensions in the outer ring. An unconstrained dimension of diameter of the outer ring was, on average, contract by $0.849 \%$ for wax pattern and 2.559\% for K4169 nickel superalloy casting. A direct linear relationship of dimensions in outer ring is established in wax pattern and its casting. The linear relationship of dimensions between wax pattern and its casting can be used to predicting distortion of casting. These results can be significantly applied to design the proper tooling allowances for ring to ring casting. The effect of wax pattern process on final casting dimensions was a small proportion in the range from $25.2 \%$ to $41.6 \%$ for diameter in outer ring. The wax pattern occupies second large changes among unconstrained dimension in casting process. We can conclude that the dimensional accuracy of castings in all casting processes is dominantly influenced by that of dimension changes in casting thermal contraction stage. The studied results are significant to evaluate the casting geometry and the key casting process factors affecting the pattern shrinkage allowances.

\section{Acknowledgements}

This research was supported by the Foundation of Shanghai University of Engineering Science (15KY0109), part by grants from Science and Technology Commission of Shanghai Municipality (No.11521100703). The supports are gratefully acknowledged.

\section{References}

[1] T.S. Piwonka, K.A. Woodburry and J.M. Wiest: Mater. Des. Vol. 21 (2000), p.366.

[2] A.S. Sabau and S. Viswanathan: Mater. Sci. Eng. A. Vol. 362 (2003), p.126.

[3] J.C. Gebelin, M.R. Jolly: J. Mater. Process. Technol. Vol. 135(2003), p. 291.

[4] R. Sadegh, R. M. Reza, A. Javad: Tsinghua. Sci. Technol. Vol. 14 (2009), p.110.

[5] Jie Zhang, Haiwei Ye: Appl. Mech. Mater. Vol. 80-81 (2011), p. 965.

[6] A.S. Sabau and S. Viswanathan: AFS. Trans. Vol. 111 (2003), p.472.

[7] D.H. Wang, B. He, F. Li and B.D. Sun: Adv. Mater. Res. Vols. 538-541 (2012), p. 1220.

[8] D.H. Wang, B. He, F. Li and B.D. Sun : Mater. Manuf. Process. Vol.28(2013), p.641. 